\title{
Deterioro cognitivo leve: ¿dónde termina el envejecimiento normal y empieza la demencia?
}

Mild cognitive impairment: where does normal ageing end and where dementia begins?

\author{
Nilton Custodio ${ }^{1,2,3}$, Eder Herrera ${ }^{4}$, David Lira ${ }^{1,2,3}$, Rosa Montesinos ${ }^{1,2,5}$, \\ Julio Linares ${ }^{1,2,5}$, Liliana Bendezú ${ }^{1,6}$ \\ 'Unidad de Diagnóstico de Deterioro Cognitivo y Prevención de Demencia, Clínica Internacional, Lima, Perú. \\ ${ }^{2}$ Unidad de Investigación, Clínica Internacional, Lima, Perú. \\ ${ }^{3}$ Servicio de Neurología, Clínica Internacional, Lima, Perú. \\ ${ }^{4}$ Universidad Nacional Mayor de San Marcos, Lima, Perú. \\ ${ }^{5}$ Servicio de Medicina Física y Rehabilitación, Clínica Internacional, Lima, Perú. \\ ${ }^{6}$ Servicio de Neurología, Hospital Militar Central, Lima, Perú.
}

\begin{abstract}
Resumen
Con el aumento de la prevalencia de demencia en el mundo, debemos poner especial atención a la evaluación diagnóstica de estadios previos a la demencia. El deterioro cognitivo leve (DCL) podría ser considerado un estadio precoz de demencia, en especial de la enfermedad de Alzheimer (AD); por lo que consideramos importante un diagnóstico adecuado, mediante la utilización de instrumentos y técnicas fiables y sensibles, que permitan discriminar entre sujetos con envejecimiento normal y patológico. Los estudios epidemiológicos muestran una elevada prevalencia de DCL en la población general. Asimismo, se ha documentado la progresión de DCL a demencia y EA, sobre todo en sujetos con DCL del tipo amnésico. La edad, el estado de portador del APOE$\varepsilon 4$, la atrofia del hipocampo en resonancia magnética y la presencia de algunos biomarcadores en líquido cefalorraquídeo parecen influir en la conversión. Se sugiere que el estudio de pacientes con sospecha de DCL sea el mismo que se emplea en la sospecha de demencia y EA. La evaluación neuropsicológica es la única prueba que permite confirmar el diagnóstico y nos ayuda a realizar una adecuada clasificación de los subtipos de DCL: amnésico, multidominio y monodominio no amnésico.
\end{abstract}

Palabras clave: Deterioro cognitivo leve, demencia, enfermedad de Alzheimer.

\section{Abstract}

Due to the increasing prevalence of dementia in the world we must put special attention to diagnostic evaluation of pre-dementia stages. Mild cognitive impairment (MCl) could be considered an early stage of dementia, particularly of Alzheimer's disease (AD), so we consider important proper diagnosis using reliable and sensitive tools and techniques in order to discriminate subjects with normal and pathological aging. Epidemiological studies show high prevalence of $\mathrm{MCl}$ in the general population as well as progression of $\mathrm{MCl}$ to dementia and $\mathrm{AD}$, especially in subjects with amnesia type $\mathrm{MCl}$. Age, APOE-E4 carrier status, hippocampus atrophy by MRI, and presence of certain biomarkers in cerebrospinal fluid can influence conversion. Evaluation of patients with $\mathrm{MCl}$ suspicion should be the same used in suspicion of dementia and AD. Neuropsychological assessment is the only test confirming diagnosis and helps making a proper classification of $\mathrm{MCl}$ subtypes: amnesic, multidomain and non-amnesic single domain.

Key words: Mild cognitive impairment, dementia, Alzheimer disease.

An Fac med. 2012;73(4):321-30

\section{INTRODUCCIÓN}

El aumento en la esperanza de vida también incrementa la prevalencia de enfermedades neurodegenerativas, siendo la demencia la de mayor impacto en la salud pública, por los elevados costos directos e indirectos que requiere su tratamiento. Según un estudio realizado en población en el Perú, la prevalencia de demencia es de 6,85\% en individuos mayores de 65 años ${ }^{(1)}$; sin embargo un gran número de adultos mayores padecen de estadios pre-demencia no diagnosticados. En la última década se ha intentado identificar los cambios cognitivos, conductuales y biológicos que ocurren durante la llamada fase 'presintomática' de la demencia. Asimismo, en la enfermedad de Alzheimer (EA) las evidencias apuntan a que el depósito gradual de la 'carga patológica' (placas de amiloide y ovillos neurofibrilares) es responsable de la disfunción cognitiva; pero, el punto desde el cual se convierte en problema para la realización de las actividades de la vida diaria, aún es controversial. La declinación cognitiva que ocurre sin cambios significativos en el funcionamiento de la vida diaria ha recibido múltiples denominaciones, siendo la más difundida deterioro cognitivo leve (DCL). El DCL es un estado intermedio entre cognición normal y demencia. No existe un evento fijo que determine el punto de inicio de la fase asintomática a la fase sintomática de la pre-demencia (2), o de la fase predemencia sintomática al inicio de la demencia ${ }^{(3)}$. Como veremos, finalmente el DCL es un trastorno heterogéneo en términos de presentación clínica, etiología y de pronóstico ${ }^{(4-6)}$. Pero, antes revisaremos aspectos epidemiológicos, los cambios cognitivos asociados a la 
edad considerados normales, y luego revisaremos aspectos de su definición, clasificación, riesgo de conversión a demencia, cuadro clínico y finalmente sugerencias para la evaluación del DCL.

\section{EPIDEMIOLOGÍA DEL DETERIORO COGNITIVO LEVE}

La valoración epidemiológica del DCL es compleja. En lo que respecta a las cifras de prevalencia, existe un amplio rango a nivel mundial. Los estudios basados en comunidad han encontrado tasas desde $1 \%$ hasta 28,3\% (tabla 1). Esta gran variabilidad se debería al tipo de muestreo (aleatorio o no), las pruebas cognitivas seleccionadas (y su punto de corte para normalidad) y la pérdida de sujetos (por abandono o fallecimiento) ${ }^{(7)}$. Además, se evidenció gran variación entre los estudios respecto a las poblaciones seleccionadas, la edad y el área de residencia (urbana o rural).

La prevalencia de DCL se incrementa con la edad, siendo $10 \%$ en individuos de 70 a 79 años y $25 \%$ en aquellos con 80 a 89 años ${ }^{(8)}$. Un estudio en afroamericanos ha estimado tasas de prevalencia de DCL de 19,2\% para el grupo de 65 a 74 años, $27,6 \%$ para los de 75 a 84 años y $38 \%$ para mayores de 85 años ${ }^{(9)}$. En términos de incidencia, las cifras publicadas varían de 5,1 a 13,7 por 1000 personas-año $^{(10,11)}$.

Otro elemento importante es la mortalidad. Diversos estudios han mostrado que los sujetos con DCL tienen una mortalidad mayor que los sujetos cognitivamente normales ${ }^{(12,13)}$. Dentro de los casos que sobrevivieron luego de 10 años de seguimiento, $27 \%$ desarrollaron demencia ( $23 \%$ EA). Por lo que consideramos que es probable que con la progresiva mayor supervivencia de la población geriátrica la proporción de afectados con DCL se incremente.

Otros estudios realizados aplicando diferentes terminologías, como 'declinación cognitiva asociada a edad', 'deterioro cognitivo, no demencia', y 'demencia mínima', han estimado tasas de prevalencia de 16 a $22 \%{ }^{(14-16)}$.

Los estudios de DCL en América Latina son escasos. Un estudio puerta a puerta en la ciudad argentina de Córdoba ha encontrado una prevalencia de $13,6 \%$ en mayores de 50 años ${ }^{(17)}$. Otro estudio poblacional en la ciudad colombiana de Medellín halló una prevalencia de DCL tipo amnésico de $9,7 \%{ }^{(18)}$. Un tercer estudio chileno comunica el seguimiento de 36 pacientes con DCL, observando la evolución a demencia en 15 de ellos en un periodo promedio de 31 meses; en un periodo de 2 años adi-

Tabla 1. Prevalencia de deterioro cognitivo leve en estudios que aplican criterios de Mayo.

\begin{tabular}{|c|c|c|c|c|c|c|}
\hline Autor & Localidad & Población & Muestra & $\begin{array}{l}\text { Criterio } \\
\text { psicométrico }\end{array}$ & Prevalencia & $\begin{array}{l}\text { Tipo de } \\
\text { estudio }\end{array}$ \\
\hline & Europa & & & & & \\
\hline Ritchie, $2001^{(23)}$ & Montpellier, Francia & NP & $833^{*}$ & NP & $>60$ años: $3,2 \%$ & Longitudinal \\
\hline Larrieu, $2002^{(24)}$ & Gironde, Francia & Comunidad & 1265 & $1,5 \mathrm{DE}$ & $\geq 65$ años: $2,8 \%$ & Longitudinal \\
\hline Hänninen, $2002^{(25)}$ & Kuopio, Finlandia & Comunidad & 806 & $1,5 \mathrm{DE}$ & $\geq 60$ años: $5,3 \%$ & Transversal \\
\hline Busse, $2003(11)$ & Leipzig, Alemania & Comunidad & $1045^{*}$ & $1 \mathrm{DE}$ & $\geq 75$ años: $3,1 \%{ }^{(a)}$ & Longitudinal \\
\hline Tognoni, $2005^{(26)}$ & Tuscania, Italia & Comunidad ${ }^{(r)}$ & 2366 & NP & $\geq 65$ años: $4,9 \%{ }^{(a)}$ & Transversal \\
\hline Consejería de Sanidad, $2005^{(27)}$ & Murcia, España & Comunidad (uyr) & 1074 & NP & $>64$ años: $4,0 \%$ & Transversal \\
\hline \multirow[t]{2}{*}{ Zanetti, $2006^{(28)}$} & Milán, Italia & Comunidad & 400 & $1 \mathrm{DE}$ (ay no-a) & $\geq 75$ años: $16,3 \%$ & Longitudinal \\
\hline & América & & & & & \\
\hline Fisk, $2003^{(29)}$ & Varios, Canadá & Comunidad (uyr) & 1790 & NP & $\begin{array}{l}\geq 75 \text { años: } 1 \%{ }^{(c)} \\
\geq 75 \text { años: } 3 \%^{(d)}\end{array}$ & Longitudinal \\
\hline López, 2003 (30) & Varios, EE.UU. & Comunidad & $2470^{*}$ & $1,5 \mathrm{DE}$ & $\geq 65$ años: $18,8 \%$ & Transversal $^{* *}$ \\
\hline Ganguli, 2004 (6) & Pensilvania, EEUU. & Comunidad ${ }^{(r)}$ & 1681 & $1 \mathrm{DE}$ & $>60$ años: $2,9 \%$ (a) & Longitudinal \\
\hline Manly, $2005^{(31)}$ & Nueva York,EE.UU. & Comunidad & $1325^{*}$ & $1,5 \mathrm{DE}$ & $\geq 65$ años: $28,3 \%$ & Longitudinal \\
\hline Mías, $2007^{(17)}$ & Córdoba, Argentina & Comunidad & 418 & $1 \mathrm{DE}$ & $\geq 50$ años: $13,6 \%$ & Transversal \\
\hline Henao-Arboleda, $2008^{(18)}$ & Medellín, Colombia & Comunidad & 848 & $1,5 \mathrm{DE}$ & > 60 años: $9,7 \%$ (a) & Transversal \\
\hline \multirow[t]{2}{*}{ Knopman, $20099^{(14)}$} & Minnesota, EE.UU. & Comunidad & 2050 & NP & $\geq 70$ años: $16,0 \%$ & Transversal \\
\hline & Asia & & & & & \\
\hline \multirow[t]{2}{*}{ Das, $2007^{(32)}$} & Kalkata, India & Comunidad & 960 & $1,5 \mathrm{DE}$ & $\geq 50$ años: $14,9 \%$ & Transversal \\
\hline & Oceanía & & & & & \\
\hline Kumar, $2005^{(33)}$ & Canberra, Queanbeyan,Australia & NP & 2551 & NP & 60-64 años: 3,7\% (a) & Transversal $^{* *}$ \\
\hline
\end{tabular}

$(\mathrm{u})=$ Población urbana; $(\mathrm{r})=$ Población rural; $\left({ }^{*}\right)=$ Muestra de sujetos no dementes; $\left({ }^{* *}\right)=$ Corte transversal en una investigación longitudinal en curso; $(\mathrm{a})=$ Aplicado para los tipos de DCL amnésico; (b)=Aplicado para los tipos de DCL multidominio y no amnésico; (c) = Aplicando definición estrictamente a los criterios de Mayo; (d) = Aplicando definición modificada, no considerando la queja subjetiva de memoria como criterio; (no-a)=Aplicado para los tipos de DCL no amnésico; (m)=Aplicado para los tipos de DCL múltiple. 
cionales, 50\%(10 casos) de los pacientes que permanecían como DCL estables en el tiempo progresaron a EA ${ }^{(19)}$.

En nuestro país no se ha realizado estudios poblacionales de incidencia o prevalencia de DCL. En la actualidad solo se cuenta con información acerca del deterioro cognitivo en el adulto mayor a partir de estudios que hallan frecuencias de deterioro cognitivo moderado y severo entre 20 y $28 \%{ }^{(20,21)}$, en pacientes geriátricos hospitalizados y ambulatorios.

Otro estudio en pacientes hospitalizados, aplicando la prueba de Pfeiffer, encontró que más de la mitad presentó algún grado de deterioro cognitivo, diferenciándose en leve, moderado y severo, con $10 \%, 12 \%$ y $24 \%$ de los casos estudiados, respectivamente ${ }^{(22)}$. Ello evidencia la elevada frecuencia del deterioro cognitivo que se sobrepone a otras patologías en pacientes hospitalizados y que muchas veces es infravalorado tanto por familiares como por el personal de salud.

\section{MEMORIA Y ENVEJECIMIENTO}

La función cognitiva de un individuo es el resultado del funcionamiento global de la percepción, atención, habilidad viso-espacial, orientación, memoria, lenguaje y cálculo, algunos de los cuales sufren un grado de deterioro variable con el envejecimiento ${ }^{(34,36)}$ :

\section{Funciones de la memoria que permanecen relativamente estables:}

a. Memoria semántica: Los hechos y el conocimiento general acerca del mundo permanecen estables, particularmente si dicha información es usada frecuentemente. Sin embargo, la recuperación de información altamente específica típicamente declina, como ocurre con los nombres ${ }^{(36,37)}$.

b. Memoria de procedimiento: Es la memoria para habilidades y hábitos, tales como manejar bicicleta o nadar; almacena información sobre cómo hacer las cosas. En general, los adultos mayores requieren más tiempo para aprender nuevas tareas ${ }^{(36,37)}$.

2. Funciones de la memoria afectadas con el envejecimiento

a. Memoria de trabajo: Comprende la tenencia y la manipulación de la información mientras se procesa otras tareas, como retener una corta lista de palabras para ordenarla alfabéticamente. También, incluye la velocidad de trabajo, memoria y aprendizaje verbal y viso-espacial, con mayor afectación de la cognición viso-espacial que la verbal ${ }^{(36,38)}$.

b. Memoria episódica: Es la memoria de los hechos de nuestras vidas individuales, de nuestras experiencias. Tanto la memoria episódica como la de trabajo son las que más se deterioran con el envejecimiento ${ }^{(39,40)}$.

c. Memoria prospectiva: Comprende la capacidad para recordar la ejecución de una acción en el futuro, como por ejemplo recordar una cita o la toma de medicación ${ }^{(36)}$.

Con la edad también declina la capacidad para recordar nueva información de texto, hacer inferencias a partir de esta, acceder a conocimientos de la memoria remota, e integrarla con nueva información de texto ${ }^{(41)}$. En términos generales, se afecta más la memoria a largo plazo que la memoria a corto plazo (con excepción de la memoria de trabajo). Respecto a las áreas cognitivas distintas a la memoria, la habilidad de focalizar la atención y realizar una tarea simple, denominada atención sostenida, se mantiene con un buen desempeño en el adulto mayor ${ }^{(9,42)}$.

\section{LENGUAJE}

El envejecimiento no parece alterar sustancialmente la capacidad de denominar objetos, aunque podría observarse confusión debido al nivel educativo bajo. Tampoco parece haber un impacto sobre la habilidad para definir palabras, reconocer la definición correcta entre las alternativas planteadas, o reconocer y corregir frases aberrantes sintácticamente. Sin embargo, se observa que los adultos mayores tienden a producir definiciones más largas, utilizando explicaciones y descripciones de muchas palabras, en comparación a

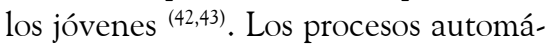
ticos sin esfuerzo consciente, el priming semántico, están preservados; mientras los procesamientos que requieren esfuerzo en su ejecución declinan con la edad. Los adultos mayores tienen una mayor dificultad tanto para procesar las estructuras sintácticas complejas como para poder hacer inferencias en la comprensión, tanto de una frase individual como en un discurso narrativo.

\section{HABILIDADES ESPACIALES}

Las habilidades espaciales hacen referencia a la capacidad de manejo del espacio corporal y extra-corporal: manejo con las diferentes partes del cuerpo, conocimiento ambiental, la cognición espacial, el mapa cognitivo, las habilidades viso-constructivas, entre otros. En el test de Wechsler, se evidencia un decremento en el ordenamiento de figuras, símbolos-dígitos y la construcción con cubos. En cuanto a las tareas de dibujo, se observa una mayor segmentación y perseverancia, un pésimo dibujo de las figuras tridimensionales e incapacidad para discriminar si lo hicieron bien ${ }^{(42)}$. Asimismo, se encuentra una disminución en el rendimiento de pruebas de reconocimiento facial y aprendizaje espacial. El adulto mayor presenta un descenso en la cognición espacial, no pudiendo visualizar un patrón complejo después de ver sus partes ${ }^{(43)}$. Finalmente, se ha reconocido también un descenso en la capacidad para identificar figuras incompletas, la codificación y recuerdo de la localización espacial, y la memoria de localización táctil ${ }^{(42,43)}$.

\section{FUNCIONES EJECUTIVAS}

Las funciones ejecutivas son habilidades vinculadas a planificación y organi- 
zación, solución de problemas nuevos (flexibilidad cognitiva), desarrollo de la planificación discursiva tanto gestual como verbal, organización de conductas, posibilidades de cambio y monitoreo de los errores, los que parecen disminuir muy levemente en el envejecimiento normal ${ }^{(42)}$.

\section{DEFINICIÓN DE DETERIORO COGNITIVO LEVE}

Se define DCL al deterioro cognitivo que supera lo normalmente esperable para la edad, pero que no cumple con criterios de demencia, pues la funcionalidad está preservada ${ }^{(44)}$. Se ha propuesto varios criterios, pero los del International Working Group on Mild Cognitive Impairment, publicados en $2001{ }^{(5)}$, son los más utilizados: 1) problemas de memoria, particularmente si son corroborados por un informante; 2) déficit de memoria objetivado mediante evaluación neuropsicológica (1,5 desviaciones estándar (DE) por debajo de lo esperado para edad y escolaridad); 3) preservación de la función cognitiva general; 4) conservación de las actividades de la vida diaria; y, 5) ausencia de demencia. Algunos investigadores no consideran la preservación de las actividades de la vida diaria como un criterio diagnóstico ${ }^{(23,29)}$. Actualmente, el National Institute on Aging-Alzheimer's Association (NIA-AA), propone al DCL como un síndrome definido por criterios clínicos, cognitivos y funcionales ${ }^{(4,45)}$; asimismo, otras causas adicionales pueden coexistir en un individuo que reúne los criterios de DCL, por lo que se pone especial énfasis a los datos clínicos, otorgando un valor adicional a los biomarcadores (beta-amiloide 42, tau/tau fosforilado en líquido cefalorraquídeo (LCR) e imágenes cerebrales), pero, secundario y dependiente de los datos clínicos ${ }^{(2)}$.

El DCL debe diferenciarse del deterioro de la memoria del envejecimiento normal, referido como 'deterioro de memoria asociado a edad' (DMAE) ${ }^{(46,47)}$. El National Institute of Mental Health (NIMH) de Estados Unidos propuso criterios diagnósticos para el DMAE: 1) edad mayor de 50 años; 2) existencia de quejas subjetivas de pérdida de memoria, descrita como gradual, sin empeoramiento brusco ni ocurrida en meses recientes, reflejadas en la vida cotidiana como dificultad para recordar nombres de personas conocidas; 3 ) rendimiento en las pruebas de memoria de por lo menos $1 \mathrm{DE}$ por debajo del promedio establecido para adultos jóvenes en la prueba estandarizada de memoria secundaria (memoria reciente); 4) conservación de las otras funciones intelectuales; y, 5) no criterios para demencia o cualquier otra condición médica que pueda causar déficit cognitivos (41,46). El DMAE hace referencia a los cambios en la memoria en adultos mayores comparados con sujetos jóvenes ${ }^{(46,48)}$. A diferencia de la memoria episódica, la memoria semántica se afecta solo en la EA y no en el envejecimiento normal ${ }^{(36,49)}$. Como el DMAE hace referencia a un déficit exclusivo de la memoria, la Asociación Internacional de Psicogeriatría y la Organización Mundial de la Salud (OMS) propusieron el término de deterioro cognitivo asociado a edad (DCAE) para caracterizar a sujetos de la tercera edad con una gama más amplia de trastornos cognitivos ya no limitados a deficiencias en la memoria ${ }^{(37,41)}$.

\section{CLASIFICACIÓN DEL DETERIORO COGNITIVO LEVE}

Petersen ha distinguido los siguientes subtipos de DCL ${ }^{(5,45)}$ :

1. DCL amnésico (DCL-a): paciente con deterioro significativo de la memoria que no cumple con criterios para demencia ${ }^{(4,5)}$. El déficit de memoria se ubica hasta 1,5 DE por debajo de la línea de normalidad ajustada para edad y escolaridad ${ }^{(35)}$. Actualmente, existe consenso que el DCL-a sería un precursor de EA ${ }^{\left({ }^{4}, 50\right)}$; sin embargo, otros procesos demenciales no atribuibles a EA también podrían causar DCL-a. Un estudio de signos parkinsonianos en pacientes con DCL encontró mayor asociación con DCL-a que con otros subtipos de DCL ${ }^{(51,52)}$.

2. DCL con déficits cognitivos múltiples o DCL multidominio (DCLmult): paciente con molestias y una funcionalidad que reflejan deficiencias leves en múltiples dominios cognitivos y conductuales, pudiendo comprometer el lenguaje, las funciones ejecutivas, viso-constructivas, o viso-espaciales ${ }^{(44,45)}$. Incluye pacientes con deterioro cognitivo entre 0,5 a $1 \mathrm{DE}$ por debajo de la línea de normalidad ajustada para edad y escolaridad. A menudo progresan hasta cumplir criterios para EA o demencia vascular (DV); una minoría de casos representa envejecimiento normal ${ }^{(45)} \mathrm{O}$, inclusive, una condición inestable con un retorno en el tiempo a la normalidad ${ }^{(53)}$.

3. DCL no amnésico de dominio único o DCL monodominio no amnésico (DCL-mnoa): paciente con deterioro aislado de algún dominio cognitivo distinto a la memoria, tal como lenguaje, funcionamiento ejecutivo o habilidades viso-espaciales ${ }^{(44)}$. En función al dominio comprometido, estos pacientes pueden progresar a otros síndromes, como afasia progresiva primaria, demencia frontotemporal, demencia con cuerpos de Lewy, DV, o enfermedad de Parkinson. Se considera que el DCL-mnoa es un estado prodrómico del grupo de demencias no Alzheimer ${ }^{(45,54)}$.

Petersen encuentra al DCL-a como el más frecuente ${ }^{(44,54)}$. Otros investigadores han señalado el siguiente orden de frecuencia: DCL-mult, DCL-a, y DCL-mnoa ${ }^{(55)}$, observándose una mayor proporción de los subtipos DCLmult y DCL-mnoa entre individuos con mayor nivel educativo ${ }^{(55,56)}$. López y col., a partir del seguimiento de pacientes con DCL plantean la existencia de dos síndromes clínicos, una forma amnésica y otra con un déficit cognitivo más amplio no amnésico, siendo este último el subtipo que se presentaría con mayor frecuencia ${ }^{(30,57)}$. 


\section{RIESGO DE CONVERSIÓN DE DETERIORO COGNITIVO LEVE A DEMENCIA}

Los pacientes con DCL tienen un riesgo incrementado de desarrollar EA ${ }^{(50)}$. Un estudio prospectivo de 798 clérigos católicos con DCL $(\mathrm{n}=211)$ y sin DCL $(n=587)$ los siguió por 4,5 años; el grupo con DCL presentó un riesgo de desarrollar EA de 3,1 (IC: 2,1 a 4,5) veces más que el grupo sin DCL ${ }^{(8)}$. Otro estudio encontró que el DCL se asoció a un riesgo de EA de 2,8 ${ }^{(58)}$. Por otro lado, las tasas de conversión de DCL a cualquier tipo de demencia han sido estimadas en 5 a $16 \%$ para la población adulta mayor ${ }^{(59-61)}$, más elevadas que las tasas de conversión a demencia en la población en general, estimadas en 1 a $3 \%$ anualmente ${ }^{(60)}$.

Estudios longitudinales han encontrado tasas de conversión de DCL a demencia y EA a los cuatro años de seguimiento de $56 \%$ (14\% anual) y $46 \%$ (11\% anual), respectivamente ${ }^{(62)}$; y, en seguimiento a seis años, se encontró progresión de hasta un $80 \%{ }^{(45)}$. El intervalo entre el diagnóstico de DCL y la conversión a demencia puede ser de hasta 8 años en algunos casos ${ }^{(63)}$. Un porcentaje variable $(11 \%$ a $40 \%)$ de pacientes con DCL mejora, e incluso se normaliza, en uno a tres años de seguimiento ${ }^{(24,59)}$.

Los factores de riesgo para conversión de DCL a demencia son diversos. La edad es el principal factor predictivo de progresión de DCL a demencia ${ }^{(64,65)}$; el género y el nivel educativo no son predictivos de conversión a demencia ${ }^{(66)}$. En una cohorte de 139 individuos con DCL, el consumo de alcohol menor a un vaso diario se asoció con una disminución en la progresión a demencia, en comparación a aquellos que se abstuvieron de consumir alcohol $(\mathrm{HR}=0,15)^{(67)}$.

Los pacientes con mayor deterioro de memoria tienen mayor probabilidad de desarrollar EA ${ }^{(68)}$. La progresión del DCL a EA se ha asociado con la presencia concomitante de síntomas psicológicos y conductuales, independien- te de APOE- $84^{\left({ }^{(69)}\right.}$. Los pacientes con DCL y ansiedad pueden tener casi el doble de riesgo de desarrollar EA en los próximos tres años comparados con los pacientes con DCL sin ansiedad (70).

Un estudio encontró que el deterioro del desempeño motor, particularmente la marcha parkinsoniana y la bradicinesia, fue asociado con conversión de DCL a EA; asimismo, los individuos con DCL tuvieron niveles de desempeño motor significativamente superiores a individuos con EA, pero inferiores a aquellos con estado cognitivo normal ${ }^{(71)}$.

Los pacientes más severamente afectados según la evaluación neuropsicológica tendrían un mayor riesgo de progresión a demencia ${ }^{(65,72)}$. Fallas en la memoria episódica y semántica podrían ser consideradas factores de riesgo para la evolución a EA ${ }^{(73,74)}$. En una cohorte anidada en que se definió la EA-prodrómica como un grupo de pacientes amnésicos al inicio del estudio que desarrollaron EA luego de dos años de seguimiento, el grupo EA-prodrómica obtuvo puntuaciones significativamente más bajas en memoria episódica y memoria semántica que el grupo de amnésicos estables que no evolucionaron a EA ${ }^{(75)}$. Además de un claro deterioro de la memoria episódica, definido en numerosos trabajos como predictivo de conversión a EA ${ }^{(73,76)}$, los pacientes presentaban también dificultades incipientes en la recuperación de datos semánticos. El deterioro de la memoria episódica en fases incipientes de la EA está relacionado con el depósito de ovillos neurofibrilares en la corteza entorrinal y el hipocampo ${ }^{(77)}$.

Algunos estudios precisan los componentes de la evaluación neuropsicológica con valor predictivo para demencia ${ }^{(78,79)}$, e incluyen el breve retraso en la memoria verbal o la memoria de reconocimiento visual, así como el compromiso de las actividades instrumentales de la vida diaria. Por otro lado, el seguimiento de los casos ha permitido concluir que probablemente los cambios intra-individuales en la función cognitiva serían más sen- sibles que la comparación con el valor de normalidad para la edad ${ }^{(80)}$. El nivel educativo se comporta como un modulador importante del rendimiento de la evaluación neuropsicológica y la

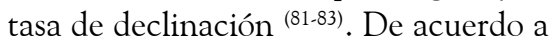
los resultados de un estudio de cohortes, la declinación cognitiva valorada mediante evaluación neuropsicológica tiene un buen valor predictivo negativo $(90 \%)$, pero pobre valor predictivo positivo $(30 \%)^{(82)}$.

El genotipo APOE- $\varepsilon 4$ ha sido asociado al riesgo de EA en la población general, pero su rol en la progresión a EA en DCL es incierto. En un estudio, sujetos con DCL portadores de APOE\&4 tuvieron mayor grado de deterioro cognitivo y atrofia del hipocampo, según resonancia magnética (RM), comparados con los no portadores ${ }^{(84)}$. Algunos estudios han encontrado que el APOE- $\varepsilon 4$ confiere un riesgo importante para la conversión de DCL hacia EA ${ }^{(85,86)}$, pero otros solo han encontrado una asociación leve o nula ${ }^{(87,88)}$.

Diversos estudios han sido orientados a buscar marcadores en LCR para predicción de conversión de DCL a demencia ${ }^{(89,90)}$, sugiriendo asociaciones, como nivel incrementado de proteína tau ${ }^{(91)}$, menor actividad de la neprilisina -enzima con capacidad para degradar el péptido beta amiloide $(A \beta)^{{ }^{(92)} \text {-, }}$ nivel disminuido de péptido beta amiloide 42 (A $\beta 42)$, baja proporción de la relación $A \beta 42 / A \beta$, baja proporción de $A \beta 42 /$ tau $^{(93-95)}$ y un nivel incrementa-

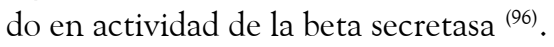

Un estudio halló la RM superior a los marcadores en LCR, tanto para determinar el estado cognitivo actual del paciente como para predecir su declinación futura; la combinación de ambas pruebas mostró una capacidad predictiva superior que al ser utilizadas de manera aislada ${ }^{(97,98)}$. La aplicabilidad en muestras poblacionales de estas observaciones es limitada, por la falta de estandarización de los métodos y la ausencia de terapéutica específica.

Estudios histopatológicos demostraron que las manifestaciones más preco- 
ces y severas de EA están localizadas en el lóbulo temporal medial (LTM), por lo que los estudios de neuroimágenes centran su atención en esta área para definir anormalidades que puedan predecir la conversión de DCL a EA ${ }^{(99)}$. Tanto el volumen cerebral como el del hipocampo en RM podrían ser predictivas de progresión de DCL a EA ${ }^{(100,101)}$. El grado como la progresión de la atrofia del LTM han sido asociadas con conversión de DCL a EA, pudiendo observarse estos cambios uno o dos años antes de la declinación cognitiva ${ }^{(102-104)}$. La edad avanzada, el APOE- 44 y un nivel cognitivo bajo basal predijeron atrofia progresiva del hipocampo en DCL ${ }^{(105)}$. Algunos estudios han asociado el DCL con la presencia de hiperintensidades en sustancia blanca subcortical y/o la presencia de infartos corticales en la RM ${ }^{(106)}$. En un estudio de 170 sujetos con DCL, este hallazgo predijo el riesgo de DV o demencia mixta en un periodo de seguimiento promedio de cuatro años ${ }^{(107) \text {. }}$

La identificación de patrones regionales de hipometabolismo cortical, usando fluorodeoxiglucosa (FDG) en las tomografías con emisión de positrones (TEP), podrían ser útiles para predecir la conversión de DCL en EA, especialmente en portadores de APOE$\varepsilon 4^{(108,109)}$. Asimismo, trazadores de amiloides para TEP, como el compuesto B de Pittsburgh marcado con C11, son herramientas prometedoras para el diagnóstico precoz de EA en pacientes con DCL ${ }^{(110,111)}$. El NIA-AA recomienda interpretar los resultados de los biomarcadores en LCR e imágenes cerebrales de acuerdo a los datos clínicos; pero, desde una perspectiva clínica, debido a que los cambios moleculares para el diagnóstico patológico de EA son también observados en angiopatía amiloide, demencia con cuerpos de Lewy o enfermedad por priones ${ }^{(2)}$.

\section{CUADRO CLÍNICO EN DETERIORO COGNITIVO LEVE}

Los pacientes con DCL manifiestan principalmente deterioro de memoria, sobre todo memoria episódica; siendo conscientes de sus deficiencias, a diferencia de lo que ocurre en EA, en que la conciencia de sus déficits está comprometida ${ }^{(54,112)}$. Estudios prospectivos y retrospectivos han demostrado que el compromiso en memoria episódica es predictivo de EA en los próximos 1 a 7 años de la evaluación inicial ${ }^{(76)}$. Ello parece indicar que los primeros cambios patológicos en EA se producen en el LTM, área crítica para memoria episódica, y consistente con la teoría de la consolidación de la memoria ${ }^{(113)}$, la cual plantea que el LTM se encarga de la codificación y recuerdo de eventos recientes; mientras que, la memoria semántica se consolida en los circuitos neocorticales, motivo por el cual se ve afectada después, en los estadios iniciales de EA ${ }^{(114)}$.

Otras molestias cognitivas, como trastornos del lenguaje (dificultad para encontrar la palabra deseada), de atención (dificultad para seguir o centrarse en una conversación), y de habilidades viso-espaciales (desorientación en entornos familiares) podrían configurar un DCL-mult, teniendo alta sensibilidad para predecir EA y DV ${ }^{(115)}$. La memoria no es la única función cognitiva afectada en los estadios pre-demencia de la EA, pues el compromiso de múltiples dominios caracteriza a la EA varios años antes del diagnóstico clínico ${ }^{(42)}$.

Los pacientes con DCL presentan frecuentemente síntomas psicológicos y conductuales (SPC), que incluyen alteraciones en conducta, afecto y síntomas psicóticos ${ }^{(116)}$. La presencia de SPC favorece la progresión del DCL a EA, disminuye la calidad de vida, exacerba las alteraciones funcionales, aumenta el desgaste del cuidador y acelera la institucionalización ${ }^{(117)}$. En el Cardiovascular Health Study (CHS) se encontró que $4,7 \%$ de pacientes con DCL tenía delusiones, $2,5 \%$ alucinaciones, $14,7 \%$ agitación/agresión y $26,3 \%$ humor depresivo, basados en el Neuropsychiatric Inventory (NPI) ${ }^{(116)}$. Östing y Skoog encontraron que hasta un 3\% de individuos no dementes mayores de 85 años de edad tenía SPC, y la psicosis fue predictiva de conversión a demencia ${ }^{(118)}$. Los pacientes con depresión simulan un cuadro clínico de DCL-mult; asimismo, las deficiencias cognitivas pueden persistir luego del tratamiento de la depresión ${ }^{(119)}$; estudios longitudinales han encontrado que el humor depresivo es un factor de riesgo para desarrollar EA ${ }^{(120,121)}$. En un ensayo clínico con 1010 pacientes con DCL, el 59\% presentó síntomas conductuales, y la depresión fue el más prevalente, seguido por irritabilidad, ansiedad, agresión y apatía; asimismo, se observó un mayor deterioro cognitivo estadísticamente significativo en aquellos que presentaron SPC respecto a quienes no los presentaron ${ }^{(122)}$. Estudios basados en la comunidad han observado un rango similar de SPC entre pacientes con DCL y EA, aunque con una mayor frecuencia y severidad en EA ${ }^{(116,123)}$.

Estudios de cohortes han hallado que la depresión y/o ansiedad están asociadas al riesgo de DCL y progresión a EA en sujetos con estado cognitivo normal y DCL, respectivamente ${ }^{(124,125)}$. Contradictoriamente, se ha observado que el deterioro cognitivo basal se asocia con un incremento de la sintomatología depresiva durante los cuatro años de seguimiento, aunque la depresión basal no se asoció con una aceleración del deterioro cognitivo ${ }^{(126)}$. Mediante el análisis de los criterios diagnósticos para DCL utilizados en seis ensayos clínicos se encontró que la exclusión de los pacientes con depresión reduciría significativamente la sensibilidad diagnóstica para los casos de DCL que desarrollarán EA ${ }^{(127)}$.

\section{EVALUACIÓN CLÍNICA DEL DETERIORO COGNITIVO LEVE}

Es importante considerar que el déficit sensorial (pérdida de agudeza visual o auditiva) y los déficit motores coexistentes en esta población pueden dificultar el diagnóstico de DCL. La evaluación de pacientes con DCL se orienta a descartar condiciones médicas tratables y determinar el estado basal, para el seguimiento. En términos generales, 
la evaluación del paciente con DCL es similar a los pacientes con sospecha de demencia, incluyendo estudios de neuroimágenes cerebrales y pruebas de sangre, para evaluar deficiencia de vitamina B12, ácido fólico e hipotiroidismo, determinar el nivel de enzimas hepáticas, función renal y hemograma completo; es necesario descartar depresión y evaluar medicamentos habituales, como antiespasmódicos, opioides, relajantes musculares, antihistamínicos, biperideno, trihexifenidilo, antidepresivos, antipsicóticos, antieméticos, ansiolíticos, antiarrítmicos y antiepilépticos, pues podrían disminuir el rendimiento cognitivo.

La evaluación neuropsicológica a cargo de un profesional con experiencia en su realización es la única prueba que permite confirmar el diagnóstico de DCL o descartarlo.

No existe consenso para una evaluación inicial de individuos con sospecha de DCL, pero se ha encontrado que las pruebas de cribado son útiles, pero no sustituyen la evaluación neuropsicológica ${ }^{(128)}$. Una vez que el paciente cumple con criterios para DCL y se establece el diagnóstico, se recomienda una evaluación neuropsicológica anual para el seguimiento cognitivo y funcional (5), aumentando la probabilidad de demencia si se evidencia declinación cognitiva, pero disminuyendo el riesgo en caso contrario ${ }^{(129)}$.

No hay criterios homogéneos para el diagnóstico de DCL mediante aplicación de pruebas neuropsicológicas. Se ha sugerido un umbral de 1,5 DE para la valoración del deterioro de memoria en las pruebas respectivas de la evaluación neuropsicológica ${ }^{(4,6)}$. En el DCL-a puede haber compromiso de otros dominios, pero usualmente no es superior a 0,5 DE. En el DCL-mult el compromiso cognitivo suele estar en el rango de 0,5 a $1 \mathrm{DE}(4,6)$.

Enseguida, revisaremos algunas pruebas neuropsicológicas:

- Evaluación basal de inteligencia: Puede incluir el subtest Vocabulario del Wechsler Adult Intelligence Scale-
III (WAIS-III) ${ }^{(130)}$ o el Wechsler Test of Adult Reading (131).

- Atención: Se puede evaluar la atención auditiva al solicitar al paciente que repita una serie de dígitos; la atención visual puede incluir recordar y correlacionar una serie de figuras; habitualmente se recomienda pruebas cortas y simples de atención sostenida, como el Trail-Making Test-A ${ }^{(132)}$.

- Función ejecutiva: La inhibición y atención selectiva puede ser evaluada con el Stroop Color-Word Interference Test ${ }^{(133)}$, la flexibilidad mental con el Trail-Making Test-B ${ }^{(132)}$. Para tareas más complejas de funciones ejecutivas, se puede utilizar el Wisconsin Card Sorting Test ${ }^{(134)}$.

- Memoria: La medición objetiva de la memoria incluye pruebas que varían en extensión y complejidad (por ejemplo, estímulos estructurados y cortos versus lista larga de palabras o figuras visuales complicadas), modalidad de presentación (auditivo o visual) y demandas lingüísticas (figuras geométricas versus palabras). Además, es importante detectar si la falla de memoria se produce en un estadio particular del proceso de aprendizaje: codificación, retención o recuerdo. Las deficiencias atencionales pueden disminuir la capacidad del individuo para codificar o recordar la información. La evaluación de la memoria verbal incluye tareas con listas de palabras, como el Rey Auditory Verbal Learning Test ${ }^{(135)} \mathrm{o}$ el California Verbal Learning Test ${ }^{(136)}$. La evaluación de la memoria no verbal puede requerir el reconocimiento o la reproducción de figuras geométricas o fotografías de rostros.

- Lenguaje: Desde el inicio de la entrevista, se puede evaluar fluencia y prosodia, así como dificultad para encontrar palabras. La denominación por confrontación puede ser evaluada con el Boston Naming Test ${ }^{(137)}$, La velocidad del recuerdo de palabras con el Controlled Oral Word Association Test ${ }^{(138)}$, que re- quiere que el individuo genere palabras en respuesta a letras (F, A y S) o categorías (animales o frutas), bajo ciertas condiciones de tiempo.

- Funciones espaciales y construccionales: La función viso-espacial incluye tareas que requieren construcción o copia de figuras con dimensión, capaces de detectar negligencias visuales. Otras tareas visuales más complejas incluye el test de figuras complejas del WAIS-III ${ }^{(130)}$. La habilidad de viso-construcción es evaluada con tareas simples, como la prueba de dibujo del reloj ${ }^{(139)} \mathrm{y}$ el dibujo del cubo ${ }^{(140)}$; sus alteraciones pueden reflejar trastornos de la planificación y ejecución motora. Otras tareas viso-construccionales más complejas incluyen el WAIS-III Block Design Test ${ }^{(130)}$ y el Rey Osterreith Complex Figure ${ }^{(141)}$.

- Humor: La evaluación del humor es un componente crítico en la evaluación del paciente con DCL, pues un trastorno emocional puede causar o exacerbar problemas cognitivos. Para una adecuada evaluación se recomienda el Beck Depression Inventory-II ${ }^{(142)}$, o la Geriatric Depression Scale ${ }^{(143)}$, aunque también se puede realizar el Neuropsychiatric Inventory ${ }^{(144)}$.

En conclusión, si bien es cierto, existen controversias respecto al punto crítico en el que se produce el compromiso funcional para determinar el inicio de la demencia, lo que no ofrece controversias es el hecho de que gran proporción de individuos con EA en estados incipientes empiezan a mostrar cambios sutiles en la cognición y conducta y tienen cambios neuropatológicos iniciados décadas atrás, lo que evidencia una enfermedad neurodegenerativa. El DCL sería un estado intermedio entre envejecimiento cognitivo normal y demencia. Asimismo, existen factores de riesgo para conversión de DCL a demencia, como son edad, estado de portador del APOE- 4 , atrofia del hipocampo en RM y presencia de biomarcadores en LCR. Teniendo en 
cuenta las diferentes estrategias de tratamiento para la modificación del curso de la enfermedad en la demencia, es indispensable que los clínicos nos preparemos para detectarla en forma precoz, pues va a ser importante para intentar disminuir la carga económica, familiar y de los servicios de salud de la demencia. En ese sentido, la evaluación neuropsicológica es indispensable para el diagnóstico del DCL, siendo también necesario interpretar los resultados clínicos, de laboratorio y las neuroimágenes, para hacer un diagnóstico correcto. En todos los pacientes es indispensable tener una evaluación neuropsicológica basal, para medir los cambios futuros; sin dejar de tomar atención a los cambios conductuales, psicológicos y funcionales. La evaluación neuropsicológica es la única prueba que puede detectar déficits cognitivos sutiles, que podrían pasar desapercibidos, sobre todo en pacientes con nivel de educación alto o en el caso de condiciones tratables. Asimismo, permitiría ayudar a determinar el subtipo de DCL y hacer el seguimiento de conversión del DCL a demencia o su remisión a la normalidad.

\section{REFERENCIAS BIBLIOGRÁFICAS}

1. Custodio N, García A, Montesinos R, Escobar J, Bendezú L. Prevalencia de demencia en una población urbana de Lima-Perú: Un estudio puerta a puerta. An Fac med. 2008;69(4):233-8.

2. Sperling R, Aisen P, Beckett L, Bennett D, Craft S, Fagan A, Iwatsubo T, et al. Towards defining the preclinical stages of Alzheimer's disease: recommendations from the National Institute on AgingAlzheimer's Association workgroups on diagnostic guidelines for Alzheimer's disease. Alzheimer's \& Dementia. 2011;7:280-92.

3. McKhann G, Knopman D, Chertkow H, Hyman B, Jack C, Cawas C, Klunk W, et al. The diagnosis of dementia due to Alzheimer's disease: recommendations from the National Institute on AgingAlzheimer's Association workgroups on diagnostic guidelines for Alzheimer's disease. Alzheimer's \& Dementia. 2011;7:263-9.

4. Petersen R, Smith G, Waring S, Ivnik R, Tangalos E, Kokmen E. Mild cognitive impairment: clinical characterization and outcome. Arch Neurol. 1999;56:303-8.

5. Petersen R, Stevens J, Ganguli M, Tangalos E, Cummings J, DeKosky S. Practice parameter: early detection of dementia: Mild cognitive impairment (an evidence-based review). Report of the Quality Standards Subcommittee of the American Academy of Neurology. Neurology. 2001;56:1133-42.

6. Ganguli M, Dodge H, Shen C, DeKosky S. Mild cognitive impairment, amnestic type: An epidemiologic study. Neurology. 2004;63:115-21.

7. Ritchie K. Mild cognitive impairment: an epidemiological perspective. Dialogues Clin Neurosci. 2004;6:401-8.

8. Bennett D, Wilson R, Schneider J, Evans D, Beckett L, Aggarwal N, Barnes L, et al. Natural history of mild cognitive impairment in older persons. Neurology. 2002;59:198-205.

9. Parks C, Decarli C, Jacoby L, Yonelinas A. Aging effects on recollection and familiarity: the role of white matter hyperintensities. Neuropsychol Dev Cogn B Aging Neuropsychol Cogn. 2010;17:42238.

10. Caracciolo B, Palmer K, Monastero R, Winblad B, Bâckman L, Fratiglioni L. Occurrence of cognitive impairment and dementia in the community: a 9 -year-long prospective study. Neurology. 2008;70:1778-85.

11. Busse A, Bischkopf J, Riedel-Heller S, Angermeyer M. Mild cognitive impairment: prevalence and incidence according to different diagnostic criteria. Results of the Leipzig Longitudinal Study of the Aged (LEILA75+). Br J Psychiatry. 2003;182:449-54.

12. Kelman H, Thomas C, Kenndy J, Cheng J. Cognitive impairment and mortality in older community residents. Am J Public Health. 1994;84:1255-60.

13. Frisoni $G$, Fratiglioni L, Fastbom J, iitanen M, Winblad B. Mortality in non demented subjects with cognitive impairment: the influence of health-related factors. Am J Epidemiol. 1999;150:1031-44.

14. Knopman D, Roberts R, Geda Y, Boeve B, Pankratz $S$, Cha R, Tangalos E, et al. Association of prior stroke with cognitive function and cognitive impairment: a population-based study. Arch Neurol. 2009;66:614-9.

15. Graham J, Rockwood K, Beattie B, Eastwood R, Gauthier S, Tuokko H, McDowell I, et al. Prevalence and severity of cognitive impairment with and without dementia in an elderly population. Lancet. 1997;349:1793-6.

16. Plassman B, Langa K, Fisher G, Heeringa S, Weir D, Ofstedal M, Burke J, et al. Prevalence of cognitive impairment without dementia in the United States. Ann Intern Med. 2008;148:427-34.

17. Mias C, Sassi M, Masih M, Querejeta A, Krawchik R. Deterioro cognitivo leve: estudio de prevalencia y factores sociodemograficos en la ciudad de Cordova, Argentina. Rev Neurol. 2007;44:733-8.

18. Henao-Arboleda E, Aguirre-Acevedo D, Muñoz C, Pineda D, Lopera F. Prevalencia de deterioro cognitivo leve de tipo amnésico en una población colombiana. Rev Neurol. 2008;46:709-13.

19. Donoso A, Behrens M, Venegas P. Deterioro cognitivo leve: seguimiento de 10 casos. Rev Chil Neuro-Psiquiat. 2003;41:117-22.

20. Villar D, Varela L, Chigne O. Evaluación geriátrica integral en pacientes hospitalizados y ambulatorios. Bol Soc Peru Med Inter. 2000;13(3):143-50.

21. Lisigurski $M$, Varela L, Ortiz P. Valoración geriátrica integral en una población de adultos mayores. Rev Soc Per Med Inter. 2002;15:39-45.

22. Varela L, Chávez H, Gálvez M, Méndez F. Caracteristicas del deterioro cognitivo en el adulto mayor hospitalizado a nivel nacional. Rev Soc Per Med Inter. 2004;17:37-42.

23. Ritchie K, Artero S, Touchon J. Classification criteria for mild cognitive impairment: A population-based validation study. Neurology. 2001;56:37-42.

24. Larrieu S, Letenneur L, Orgogozo J, Fabrigoule C, Amieva H, Le Carret N, Barberger-Gateau P, et al. Incidence and outcome of mild cognitive impairment in a population-based prospective cohort. Neurology. 2002;59:1594-9.

25. Hänninen T, Hallikainen M, Tuomainen S, Vanhanen
$\mathrm{M}$, Soininen $\mathrm{H}$. Prevalence of mild cognitive impairment: a population-based study in elderly subjects. Acta Neurol Scand. 2002;106:148-54.

26. Tognoni G, Ceravolo R, Nucciarone B, Bianchi F, Del'Agnello G, Ghicopulos I, Siciliano G, et al. From mild cognitive impairment to dementia: a prevalence study in a district of Tuscany, Italy. Acta Neurol Scand. 2005;112:65-71.

27. España, Consejería de Sanidad. Prevalencia de demencia y deterioro cognitivo en la Región de Murcia. Murcia, 2005. [página de internet] Murcia, España, Consejería de Sanidad; 2005. [citado setiembre 2011] Disponible en: www.murciasalud. es/archivo. php?id=80076.

28. Zanetti M, Ballabio C, Abbate C, Cutaia C, Vergani C, Bergamaschini L. Mild cognitive impairment subtypes and vascular dementia in communitydwelling elderly people: a 3-year follow-up study. J Am Geriatr Soc. 2006;54:580-6.

29. Fisk J, Merry H, Rockwood K. Variations in case definition affect prevalence but not outcomes of mild cognitive impairment. Neurology. 2003;61:1179-84.

30. Lopez O, Jagust W, DeKosky S, Becker J, Fitzpatrick A, Dulberg C, Breitner J, et al. Prevalence and classification of mild cognitive impairment in the Cardiovascular Health Study Cognition Study: part 1. Arch Neurol. 2003;60:1385-9.

31. Manly J, Bell-McGinty S, Tang M, Schupf N, Stern $Y$, Mayeux R. Implementing diagnostic criteria and estimating frequency of mild cognitive impairment in an urban community. Arch Neurol. 2005;62:1739-46.

32. Das S, Bose P, Biswas A, Dutt A, Banerjee T, Hazra $A$, Raut $D$, et al. An epidemiologic study of mild cognitive impairment in Kolkata, India. Neurology. 2007;68:2019-26.

33. Kumar R, Dear K, Christensen H, Ilschner S, Jorm A, Meslin C, Rosenman S, et al. Prevalence of mild cognitive impairment in 60- to 64-year-old community-dwelling individuals: The Personality and Total Health through Life 60+ Study. Dement Geriatr Cogn Disord. 2005;19:67-74.

34. Bennett D, Schneider J, Arvanitakis Z, Kelly J, Aggarwal N, Shah R, Wilson R. Neuropathology of older persons without cognitive impairment from two community-based studies. Neurology. 2006;66:1837-44.

35. Albert M, Moss M, Blacker D, Tanzi R, McArdle J. Longitudinal change in cognitive performance among individuals with mild cognitive impairment. Neuropsychol. 2007;21:158-69.

36. Luo L, Craik F. Aging and memory: a cognitive approach. Can J Psychiatry. 2008;53:346-53.

37. Crook T, Bartus R, Ferris S, Whitehouse P, Cohen G, Gershon S. Age-associated memory impairment: proposed diagnostic criteria and measures of clinical change: report of a National Institute of Mental Health Work Group. Dev Neuropsychol. 1986;2:261-76.

38. Jenkins L, Myerson J, Joerding J, Hale S. Converging evidence that visuospatial cognition is more age-sensitive than verbal cognition. Psychol Aging. 2000;15:157-75.

39. Head D, Rodrigue K, Kennedy K, Raz N. Neuroanatomical and cognitive mediators of agerelated differences in episodic memory. Neuropsychol. 2008;22:491-507.

40. Bäckman L, Small B, Fratiglioni L. Stability of the preclinical episodic memory deficit in Alzheimer's disease. Brain. 2001;124:96-102.

41. Hannon B, Daneman M. Age-related changes in reading comprehension: an individual-differences perspective. Exp Aging Res. 2009;35:432-56. 
42. Bäckman L, Jones S, Berger A, Laukka E, Small B. Cognitive impairment in preclinical Alzheimer's disease: A meta-analysis. Neuropsychol. 2005;19:520-31.

43. Bondy M, Jak A, Delano-Wood L, Jacobson M, Delis D, Salmon D. Neuropsychological contributions to the early identification of Alzheimer's disease. Neuropsychol. 2008;18:73-90.

44. Petersen R. Conceptual overview. En: Mild Cognitive Impairment: Aging to Alzheimer's Disease, 1st Ed, New York: Oxford University Press, 2003:1-14.

45. Petersen R. Mild cognitive impairment as a diagnostic entity. J Intern Med. 2004;256:183-94.

46. Miller S, Mitchell M, Woodard J, Davey A, Martin P, Poon L; for the Georgia Centenarian Study. Cognitive perfomance in centenerians and the oldest old: Norms of the Georgia Centenarian study. Aging Neuropsychol Cognition. 2010;17:575-90.

47. Levy R. Aging-associated cognitive decline. Working Party of the International Psychogeriatric Association in collaboration with the World Health Organization. Int Psychogeriatr. 1994;6:63-8.

48. Smith G, Ivnik J, Petersen C, Malec J, Kokmen E, Tangalos E. Age-associated memory impairment diagnoses: Problems of reliability and concerns for terminology. Psychol Aging. 1991;6:551-8.

49. Bondi M, Monsch A, Galasko D, Butters N, Salmon D, Delis D. Preclinical cognitive markers of dementia of the Alzheimer type. Neuropsychol. 1994;8:374-84.

50. Morris J, Storandt M, Miller J, McKell D, Price $\mathrm{J}$, Rubin E. Mild cognitive impairment represents early-stage Alzheimer disease. Arch Neurol. 2001;58:397-405.

51. Boyle P, Wilson R, Aggarwal N, Arvanitakis Z, Kelly J, Bienias J, Bennett D. Parkinsonian signs in subjects with mild cognitive impairment. Neurology. 2005;65:1901-6.

52. Louis E, Schupf N, Manly J, Marder K, Tang M, Mayeux R. Association between mild parkinsonian signs and mild cognitive impairment in a community. Neurology. 2005;64:1157-61

53. Salmon D, Hodges J. Introduction: mild cognitive impairment--cognitive, behavioral, and biological factors. Neurocase. 2005;11:1-2.

54. Petersen R. Mild cognitive impairment. Continuum Lifelong Learning in Neurology. 2007:13:15-38.

55. Markesbery W, Schmitt F, Kryscio R, Davis D, Smith C, Wekstein D. Neuropathologic substrate of mild cognitive impairment. Arch Neurol. 2006;63:3846.

56. Busse A, Hensel A, Gühne U, Angermeyer MC, Riedel-Heller S. Mild cognitive impairment: longterm course of four clinical subtypes. Neurology. 2006;67:2176-85.

57. Lopez O, Kuller L, Becker J, Dulberg C, Sweet R, Gach M, DeKosky S. Incidence of dementia in mild cognitive impairment in the cardiovascular health study cognition study. Arch Neurol. 2007;64:416-20

58. Manly J, Tang M, Schupf N, Stern Y, Vonsattel J, Mayeux R. Frequency and course of mild cognitive impairment in a multiethnic community. Ann Neurol. 2008;63:494-506.

59. Palmer K, Wang H, Backman L, Winblad B, Fratiglioni L. Differential evolution of cognitive impairment in nondemented older persons: results from the Kungsholmen Project. Am J Psychiatry. 2002; 159:436-442.

60. Boyle P, Wilson R, Aggarwal N, Tang Y, Bennett D. Mild cognitive impairment: risk of Alzheimer disease and rate of cognitive decline. Neurology. 2006;67:441-5.
61. Fischer $P$, Jungwirth $S$, Zehetmayer $S$, Weissgram S, Hoenigschnabl S, Gelpi E, Krampla W, et al. Conversion from subtypes of mild cognitive impairment to Alzheimer dementia. Neurology. 2007;68:288-91.

62. Rountree S, Waring S, Chan W, Lupo P, Darby E, Doody R. Importance of subtle amnestic and nonamnestic deficits in mild cognitive impairment: prognosis and conversion to dementia. Dement Geriatr Cogn Disord. 2007;24:476-82.

63. Stokholm J, Jakobsen O, Czarna J, Mortensen H, Waldemar $G$. Years of severe and isolated amnesia can precede the development of dementia in early-onset Alzheimer's disease. Neurocase. 2005;11:48-55.

64. Kryscio R, Schmitt F, Salazar J, Mendiondo M, Markesbery $W$. Risk factors for transitions from normal to mild cognitive impairment and dementia. Neurology. 2006;66:828-32.

65. Amieva H, Letenneur L, Dartigues J, Rouch-Leroye I, Sourgen C, D'Alchée-Birée F, Dib M, et al. Annual rate and predictors of conversion to dementia in subjects presenting mild cognitive impairment criteria defined according to a population-based study. Dement Geriatr Cogn Disord. 2004;18:87-93.

66. DeCarli C, Mungas D, Harvey D, Reed B, Weine $\mathrm{M}$, Chui $\mathrm{H}$, Jagust W. Memory impairment, but not cerebrovascular disease, predicts progression of $\mathrm{MCl}$ to dementia. Neurology. 2004;63:220-7.

67. Solfrizzi V, D'Introno A, Colacicco A, Capurso C Del Parigi A, Baldassarre C, Scapicchio P, et al. Alcohol consumption, mild cognitive impairment, and progression to dementia. Neurology. 2007; 68:1790-9

68. Hunderfund A, Roberts R, Slusser T, Leibson C Geda Y, Ivnik R, Tangalos E, et al. Mortality in amnestic mild cognitive impairment: a prospective community study. Neurology. 2006;67:1764-8.

69. Steffens D, Plassman B, Helms M, Welsh-Bohme K, Saunders A, Breitner J. A twin study of lateonset depression and apolipoprotein E $\mathcal{4} 4$ as risk factors for Alzheimer's disease. Biol Psychiatry. 1997;41:851-6.

70. Dal Forno G, Palermo M, Donohue J, Karagiozis H, Zonderman A, Kawas C. Depressive symptoms, sex, and risk for Alzheimer's disease. Ann Neurol. 2005;57:381-7.

71. Aggarwal N, Wilson R, Beck T, Bienias J, Bennett $D$. Motor dysfunction in mild cognitive impairment and the risk of incident Alzheimer disease. Arch Neurol. 2006;63:1763-9.

72. Fleisher A, Sowell B, Taylor C, Gamst A, Petersen R, Thal L; the Alzheimer's Disease Cooperative Study. Clinical predictors of progression to Alzheimer disease in amnestic mild cognitive impairment. Neurology. 2007;68:1588-95.

73. Sarazin M, Berr C, De Rotrou J, Fabrigoule C Pasquier F, Legrain S, Michel B, et al. Amnestic syndrome of the medial temporal type identifies prodromal AD: a longitudinal study. Neurology. 2007;69:1859-67.

74. Frisoni G, Galluzzi S, Signorini M, Garibotto V, Paghera B, Binetti G, Canu E, et al. Preliminary evidence of validity of the revised criteria for Alzheime disease diagnosis: report of 2 cases. Alzheimer Dis Assoc Disord. 2010;24:108-14.

75. Molinuevo J, Gomez-Anson B, Monte G, Bosch B, Sánchez-Valle R, Rami L. Neuropsychological profile of prodromal Alzheimer's disease (Prd-AD) and their radiological correlates. Arch Gerontol Geriatr. 2010;52:190-6.

76. Tounsi H, Deweer B, Ergis A, Van der Linden M Pillon B, Michon A, Dubois B. Sensitivity to semantic cuing: an index of episodic memory dysfunction in early Alzheimer disease. Alzheimer Dis Assoc Disord. 1999;13:38-46.

77. Braak H, Braak E. Neuropathological staging of Alzheimer related changes. Acta Neuropathol. 1991;82:239-59.

78. Chen P, Ratcliff G, Belle S, Cauley J, Dekosky S, Ganguli M. Cognitive tests that best discriminate between presymptomatic $A D$ and those who remain nondemented. Neurology. 2000;55:1847-53.

79. Aggarwal N, Wilson R, Beck T, Bienias J, Bennett D. Mild cognitive impairment in different functional domains and incident Alzheimer's disease. J Neurol Neurosurg Psychiatry. 2005;76:1479-84.

80. Storandt M, Grant E, Miller J, Morris J. Longitudinal course and neuropathologic outcomes in original vs revised $\mathrm{MCl}$ and in pre-MCl. Neurology. 2006;67:467-73.

81. Touchon J, Ritchie K. Prodromal cognitive disorder in Alzheimer's disease. Int J Geriatr Psychiatry. 1999;14:556-63.

82. Sacuiu S, Sjögren M, Johansson B, Gustafson D, Skoog I, Prodromal cognitive signs of dementia in 85-year-olds using four sources of information. Neurology. 2005;65:1894-900.

83. Laukka E, MacDonald S, Backman L. Contrasting cognitive trajectories of impending death and preclinical dementia in the very old. Neurology. 2006;66:833-8.

84. Farlow M, He Y, Tekin S, Xu J, Lane R, Charles $\mathrm{H}$. Impact of APOE in mild cognitive impairment. Neurology. 2004;63:1898-901.

85. Buerger K, Teipel S, Zinkowski R, Blennow K, Arai $\mathrm{H}$, Engel R, Hofmann-Kiefer $\mathrm{K}$, et al. CSF tau protein phosphorylated at threonine 231 correlates with cognitive decline in $\mathrm{MCl}$ subjects. Neurology. 2002;59:627-9.

86. Petersen R, Smith G, Ivnik R, Tangalos E, Schaid D, Thibodeau S, Kokmen E, et al. Apolipoprotein E status as a predictor of the development of Alzheimer's disease in memory-impaired individuals. JAMA. 1995;273:1274-8.

87. Devanand D, Pelton G, Zamora D, Liu X, Tabert M, Goodkind M, Scarmeas N, et al. Predictive utility of apolipoprotein E genotype for Alzheimer disease in outpatients with mild cognitive impairment. Arch Neurol. 2005:62:975-80.

88. Aggarwal N, Wilson R, Beck T, Bienias J, BerryKravis E, Bennett D. The apolipoprotein E epsilon4 allele and incident Alzheimer's disease in persons with mild cognitive impairment. Neurocase. 2005;11:3-7

89. Riemenschneider M, Lautenschlager N, Wagenpfeil S, Diehl J, Drzezga A, Kurz A. Cerebrospinal fluid tau and beta-amyloid 42 proteins identify Alzheimer disease in subjects with mild cognitive impairment. Arch Neurol. 2002;59:1729-34.

90. Simonsen A, McGuire J, Hansson O, Zetterberg H, Podust V, Davies H, Waldemar G, et al. Novel panel of cerebrospinal fluid biomarkers for the prediction of progression to Alzheimer dementia in patients with mild cognitive impairment. Arch Neurol. 2007;64:366-70.

91. Mitchell A. CSF phosphorylated tau in the diagnosis and prognosis of mild cognitive impairment and Alzheimer's disease: a meta-analysis of 51 studies. J Neurol Neurosurg Psychiatry. 2009;80:966-75.

92. Maruyama M, Higuchi M, Takaki Y, Matsuba Y, Tanji H, Nemoto M, Tomita N, et al. Cerebrospinal fluid neprilysin is reduced in prodromal Alzheimer's disease. Ann Neurol. 2005;57:832-42.

93. Li G, Sokal I, Quinn J, Leverenz J, Brodey M, Schellenberg G, Kaye J, et al. CSF tau/Abeta42 ratio for increased risk of mild cognitive impairment: a follow-up study. Neurology. 2007;69:631-9. 
94. Fagan A, Head D, Shah A, Marcus D, Mintum M, Morris J, Holtzman D. Decreased cerebrospinal fluid Abeta(42) correlates with brain atrophy in cognitively normal elderly. Ann Neurol. 2009;65:176-83.

95. Visser P, Verhey F, Knol D, Scheltens P, Wahlund L, Freund-Levi Y, Tsolaki M, et al. Prevalence and prognostic value of CSF markers of Alzheimer's disease pathology in patients with subjective cognitive impairment or mild cognitive impairment in the DESCRIPA study: a prospective cohort study. Lancet Neurol. 2009;8:619-27.

96. Zhong Z, Ewers M, Teipel S, Bürger K, Wallin A, Blennow K, He P, et al. Levels of beta-secretase (BACE1) in cerebrospinal fluid as a predictor of risk in mild cognitive impairment. Arch Gen Psychiatry. 2007;64:718-26.

97. Vemuri P, Wiste H, Weigand S, Shaw L, Trojanowski J, Weiner M, Knopman D, et al. MRI and CSF biomarkers in normal, $\mathrm{MCl}$, and $\mathrm{AD}$ subjects: diag nostic discrimination and cognitive correlations. Neurology. 2009;73:287-93

98. Vemuri $\mathrm{P}$, Wiste $H$, Weigand $S$, Shaw L, Trojanowski J, Weiner M, Knopman D, et al. MRI and CSF biomarkers in normal, $\mathrm{MCl}$, and $\mathrm{AD}$ subjects: predicting future clinical change. Neurology. 2009;73:294-301.

99. van de Pol A, Korf E, van der Flier W, Brashear R, Fox N, Barkhof F, Scheltens P. Magnetic resonance imaging predictors of cognition in mild cognitive impairment. Arch Neurol. 2007;64:1023-8.

100. Stewart R, Dufouil C, Godin O, Ritchie K, Maillard $P$, Delcroix N, Crivello F, et al. Neuroimaging correlates of subjective memory deficits in a community population. Neurology. 2008;70:1601-7.

101. Zimmerman M, Pan J, Hetherington H, Katz M, Verghese J, Buschke H, Derby C, et al. Hippocampal neurochemistry, neuromorphometry, and verbal memory in nondemented older adults. Neurology. 2008;70:1594-600.

102. Fleisher A, Sun S, Taylor C, Ward C, Gamst A, Petersen R, Jack Jr C, et al. Volumetric MRI vs clinical predictors of Alzheimer disease in mild cognitive impairment. Neurology. 2008;70:191-9.

103. Mungas D, Harvey D, Reed B, Jagust W, DeCarli C, Beckett L, Mack W, et al. Longitudinal volumetric $\mathrm{MRI}$ change and rate of cognitive decline. Neurology. 2005;65:565-71.

104. Erten-Lyons D, Howieson D, Moore M, Quinn J, Sexton $\mathrm{G}$, Silbert L, Kaye J. Brain volume loss in MC predicts dementia. Neurology. 2006;66:233-5.

105. van de Pol L, van der Flier W, Korf E, Fox N, Barkhof $F$, Scheltens $P$. Baseline predictors of rates of hippocampal atrophy in mild cognitive impairment. Neurology. 2007;69:1491-7.

106. Debette S, Bombois S, Bruandet A, Delbeuk X, Lepoittevin S, Delmaire C, Leys D, et al. Subcortical hyperintensities are associated with cognitive decline in patients with mild cognitive impairment. Stroke. 2007;38:2924-30.

107. Bombois S, Debette S, Bruandet A, Delbeuk X Delmaire C, Leys D, Pasquier F. Vascular subcortical hyperintensities predict conversion to vascula and mixed dementia in $\mathrm{MCl}$ patients. Stroke. 2008;39:2046-51.

108. Mosconi L, Perani D, Sorbi S, Herholz K, Nacmias B, Holthoff $\bigvee$, Salmon $E$, et al. MCl conversion to dementia and the APOE genotype: A prediction study with FDG-PET. Neurology. 2004;63:2332-40.

109. Chetelat G, Desgranges B, de la Sayette V, Viadet F, Eustache F, Baron J. Mild cognitive impairment: Can FDG-PET predict who is to rapidly convert to Alzheimer's disease? Neurology. 2003;60:1374-7.
110. Wolk D, Price J, Saxton J, Snitz B, James J, Lopez $\mathrm{O}$, Aizenstein $\mathrm{H}$, et al. Amyloid imaging in mild cognitive impairment subtypes. Ann Neurol. 2009;65:557-68.

111. Okello A, Koivunen J, Edison $\mathrm{P}$, Archer H, Turkheimer F, Nagren K, Bullock R, et al. Conversion of amyloid positive and negative $\mathrm{MCl}$ to $\mathrm{AD}$ over 3 years: an 11C-PIB PET study. Neurology. 2009;73:754-60.

112. Petersen R, Doody R, Kurz A, Mohs R, Morris $J$, Rabins P, Ritchie K, et al. Current concepts in mild cognitive impairment. Arch Neurol. 2001;58:1985-92.

113. Squire L, Stark C, Clark R. The Medial Temporal Lobe. Annu Rev Neurosci. 2004;27:279-306.

114. Wiltgen B, Brown R, Talton L, Silva A. New circuits for old memories: the role of the neocortex in consolidation. Neuron. 2004;44:101-8.

115. Rasquin S, Lodder J, Visser P, Lousberg R, Verhey $\mathrm{F}$. Predictive accuracy of $\mathrm{MCl}$ subtypes for Alzheimer's disease and vascular dementia in subjects with Mild Cognitive Impairment: a 2-year follow-up study. Dement Geriatr Cogn Disord. 2005;19:113-9.

116. Lyketsos C, Lopez O, Jones B, Fitzpatrick A, Breitner J, DeKosky S. Prevalence of neuropsychiatric symptoms in dementia and mild cognitive impairment: results from the Cardiovascular Health Study. JAMA. 2002;288:1475-83.

117. Teng E, Lu P, Cummings J. Neuropsychiatric symptoms are associated with progression from mild cognitive impairment to Alzheimer's disease. Dement Geriat Cogn Disord. 2007:24:253-9.

118. Östling S, Skoog I. Psychotic symptoms and paranoid ideation in a nondemented population based sample of the very old. Arch Gen Psychiatry. 2002;59:53-9.

119. Butters M, Becker J, Nebes R, Zmuda M, Mulsant B, Pollock B, Reynolds C. Changes in cognitive functioning following treatment of late-life depression. Am J Psychiatry. 2000;157:1949-54.

120. Wilson R, Barnes L, Mendes de Leon C, Aggarwal N, Schneider J, Bach J, Pilat J, et al. Depressive symptoms, cognitive decline and risk of AD in older persons. Neurology. 2002;59:364-70.

121. Green R, Cupples L, Kurz A, Auerbach S, Go R, Sadovnick D, Duara R, et al. Depression as a risk factor for Alzheimer's disease. The MIRAGE Study. Arch Neurol. 2003;60:753-9.

122. Feldman $H$, Scheltens $P$, Scarpini E, Hermann $N$, Mesenbrink P, Mancione L, Tekin S, et al. Behavioral symptoms in mild cognitive impairment. Neurology. 2004;62:1199-201.

123. Lopez O, Becker J, Sweet R. Non-cognitive symptoms in mild cognitive impairment subjects. Neurocase. 2005;11:65-71

124. Palmer K, Berger A, Monastero R, Winblad B, Bäc$\mathrm{kman}$ L, Fratiglioni L. Predictors of progression from mild cognitive impairment to Alzheimer disease. Neurology. 2007;68:1596-602.

125. Wilson R, Schneider J, Boyle P, Arnold S, Tang Y, Bennett D. Chronic distress and incidence of mild cognitive impairment. Neurology. 2007;68:208592.

126. Vinkers D, Gussekloo J, Stek M, Westendorp G, van der Mast R. Temporal relation between depression and cognitive impairment in old age: prospective population based study. BMJ. 2004;329:881-4.

127. Visser $P$, Scheltens $P$, Verhey F. Do $\mathrm{MCl}$ criteria in drug trials accurately identify subjects with predementia Alzheimer's disease? J Neurol Neurosurg Psychiatry. 2005;76:1348-54

128. Rami L, Bosch B, Valls-Pedret C, Caprile C, Sánchez-Valle R, Molinuevo J. Validez discriminativa y asociación del test minimental (MMSE) y del test de alteración de la memoria (T@M) con una batería neuropsicológica en pacientes con deterioro cognitivo leve amnésico y enfermedad de Alzheimer. Rev Neurol. 2009;49:169-74.

129. Triebel K, Martin R, Griffith H, Marceaux J, Okonkwo $\mathrm{O}$, Harrell L, Clark D, et al. Declining financial capacity in mild cognitive impairment: A 1-year longitudinal study. Neurology. 2009;73:928-34.

130. WAIS-III/WMS-III: Updated Technical Manual. San Antonio, Tex: The Psychological Corporation; 2002.

131. Wechsler Test of Adult Reading Manual. San Antonio, Tex: The Psychological Corporation; 2001.

132. Reitan R. The relation of the Trail-Making Test to organic brain damage. J Consult Psychol. 1955;19:393-4.

133. Stroop J. Studies of interference in serial verbal reaction. J Exp Psychol. 1935;18:643-62.

134. Grant D, Berg E. A behavioral analysis of degree of impairment and ease of shifting to new responses in a Weigl-type card sorting problem. J Exp Psychol. 1948;39:404-11.

135. Rey A. L'examen Clinique en Psychologie. Paris, France: Presses Universitaires de France; 1958.

136. Delis D, Kramer J, Kaplan E, Ober B. California Verbal Learning Test. San Antonio, Tex: The Psychological Corporation; 1987.

137. Kaplan E, Goodglass H, Weintraub S. The Boston Naming Test. 2nd ed. Philadelphia, Penn: Lippin cott, Williams \& Wilkins; 2001.

138. Ruff R, Light R, Parker S, Levin H. Benton Controlled Oral Word Association Test: reliability and updated norms. Arch Clin Neuropsychol. 1996;11:329-38.

139. Freedman M, Leech L, Kaplan E. Clock Drawing: A Neuropsychological Analysis. New York, NY: Oxford University Press; 1994.

140. Strub RL, Black FW. The Mental Status Examination in Neurology. 4th ed. Philadelphia, Penn: F.A Davis; 2000.

141. Rey A. L'examen Psychologique dans les cas D'encephapothie Traumatique. Archives de Psychologie. 1941;28:286-340.

142. Beck A, Steer R, Brown G. Beck Depression Inventory. 2nd ed. San Antonio, Tex: The Psychological Corporation; 1996.

143. Brink T, Yesavage J, Lum O, Heersema P, Adey M, Rose T. Screening tests for geriatric depression. Clin Gerontol. 1982;1:37-43.

144. Kaufer D, Cummings J, Ketchel P, Smith V, MacMillan A, Shelley T, Lopez O, et al. Validation of the NPI-Q: a brief clinical form of the Neuropsychiatric Inventory. J Neuropsychiatry Clin Neurosci. 2000;12:233-9.

Artículo recibido el 28 de mayo de 2012 y aceptado para publicación el 24 de junio de 2012.

\section{Fuente de Financiamiento: Ninguno}

Conflictos de Interés: Ninguno

Autor corresponsal:

Nilton Custodio

Servicio de Neurología, Clínica Internacional

Garcilaso de la Vega 1420

Cercado de Lima, Lima 1, Perú

Telf/fax: (511) 4319677

Correo electrónico: niltoncustodio@neuroconsultas.

com 